\title{
MACROERGONOMÍA: MODELO SISTÉMICO ORGANIZACIONAL
}

\author{
MACROERGONOMICS: ORGANIZATIONAL SYSTEMIC MODEL
}

\author{
Walter Daniel Amado ${ }^{1}$
}

\begin{abstract}
Resumen: Después de 20 años de dedicación a la consultoría de ergonomía ocupacional en grandes empresas, se esboza un modelo bajo el nombre de "Modelo Holos", cuyo objetivo será dotar a las organizaciones de una nueva cosmovisión de ergonomía, seguridad y salud ocupacional. El modelo es aplicado en una empresa alimenticia multinacional, donde en el año 2019 se culminó con la fase I de análisis cualitativo (108 preguntas), arrojando cincuenta y una oportunidades de mejora del sistema, y la fase II de análisis prospectivo, visualizando y generando veinte escenarios futuros de anticipación. En este momento, el modelo se encuentra en la fase III de análisis de propiedades emergentes. La propiedad exógena emergente de la fase III de 2020 es la COVID-19. En esta etapa se analiza cómo, dicha perturbación impacta al modelo, y poder así, determinar su grado de vulnerabilidad y resiliencia.
\end{abstract}

Palabras clave: Seguridad y salud ocupacional, ergonomía, modelo organizacional.

\begin{abstract}
After 20 years of dedication to occupational ergonomics consulting in large companies, a model is outlined under the name of "Holos Model", whose objective will be to provide organizations with a new worldview of occupational ergonomics, safety and health. The model is applied in a multinational food company, where in 2019 phase I of qualitative analysis (108 questions) was completed, yielding fiftyone opportunities to improve the system, and the phase II of prospective analysis, visualizing and generating twenty future scenarios of anticipation. At this time, the model is in phase III of analysis of emergent properties. The emerging exogenous property of phase III of 2020 is COVID 19. In this stage, it is analyzed how said disturbance impact the model, and thus be able to determine its degree of vulnerability and resilience.
\end{abstract}

Keywords: Occupational safety and health, ergonomics, organizational model.

Recepción: 03.08.2020 / Revisión: 12.10.2020 / Aceptación: 29.10.2020

\section{Introducción}

Muchas veces los profesionales de la seguridad y salud ocupacional se preguntan cómo transformar los puestos y entornos de trabajo, logrando el tan ansiado bienestar de los trabajadores y a su vez, obtener el mayor rendimiento general del sistema para las organizaciones. En base a esta lógica, la fórmula planteada sería: más salud y más producción. Fórmula que obedece al cálculo ganar-ganar de todos los actores del sistema.

${ }^{1}$ Universidad Católica de La Plata. Buenos Aires, Argentina. Correo electrónico: wamado@me.com. Orcid: https://orcid.org/oooo-0002-4110-2711 
Ahora bien, la experiencia indica que muchas organizaciones operan con fórmulas distintas. El enfoque económico mundial imperante genera la necesidad de contar con una mayor producción y en algunos casos no se considerará la salud y el bienestar de los trabajadores. Cabe destacar la importancia, control y cumplimiento de la legislación en la materia, como reguladora y equilibrante de este sistema.

Por lo expuesto, se hace relevante contar con una mirada holística del mundo del trabajo y de las organizaciones. Esta perspectiva ayudará a entender y decodificar el mapa organizacional desde un nivel macro, para ir descendiendo al nivel micro operacional del sistema humano-máquina.

Con la aplicación del "Modelo Holos", se abandona la arraigada observación de las organizaciones como estructuras compartimentadas y se abraza un nuevo enfoque integral y sistémico, atendiendo no sólo a la acción y reacción directa de sus elementos, sino también a sus interrelaciones. El objetivo es dotar y dar a conocer un modelo de aplicación que busca el rendimiento general del sistema y a su vez el bienestar de los trabajadores. Entiéndase por rendimiento general del sistema a la optimización de la productividad, calidad, innovación, flexibilidad, confiabilidad, sostenibilidad, entre otros y por bienestar de los trabajadores, al logro de una mayor seguridad laboral, salud, satisfacción, placer, aprendizaje, desarrollo personal, etc.

\section{Materiales y métodos}

Para diseñar este tipo de intervención se trabajó en un modelo de aplicación, al que denominamos "Modelo Holos", cuyos sistemas, subsistemas y actores fueron concebidos por un equipo de raíz interdisciplinaria de una empresa alimenticia multinacional, más el soporte y andamiaje teórico de docentes e investigadores de la Universidad Nacional de Tres de Febrero y de la Facultad Católica de La Plata.

La fase I de este modelo fue llevada a cabo durante el año 2019, con el éxito esperado por la empresa. Una vez terminada esta fase, se detectaron importantes oportunidades de mejora las cuales se aplicarán durante el corriente año. La fase II implica un análisis "prospectivo" de los veintisiete subsistemas, más el agregado de una fase III de análisis de "propiedades emergentes."

En la cátedra de ergonomía de la licenciatura en higiene y seguridad del trabajo, hace años que los alumnos aplican con éxito este modelo, el cual los ayuda a comprender y conciliar la mirada micro y macro de la ergonomía.

Con esta aplicación tanto en el ámbito privado de las empresas como en el aula, se aprecia la potencia del trabajo en equipo e interdisciplinario. Con un buen modelo y un noble equipo se pueden lograr grandes cosas. 


\section{Resultados y discusión}

Dicho modelo es aplicado en tres fases y bajo un enfoque de tipo sistémico. Para comenzar y dar sustento conceptual al mismo, primero se debe dilucidar qué es una organización y qué es un sistema. Una organización es: a) la configuración de un grupo humano complejo; b) que actúa dentro de un contexto tempero espacial concreto, artificial y deliberadamente constituido; c) para la realización de fines y necesidades específicas (Filippi, 2003). A su vez, un sistema es un conjunto complejo de partes interdependientes que interactúan para adaptarse a un entorno constantemente cambiante, con el fin de lograr sus objetivos. Todas las partes del sistema dependen de las otras. Cualquier cambio o influencia sobre un componente afecta inevitablemente los otros componentes del sistema. Cada sistema recibe recursos, o entradas, de su entorno, las procesa y exporta productos o salidas. Las partes internas del sistema que realizan el procesamiento activo de entradas en salidas se conocen como subsistemas. Cada sistema es en sí una parte de un sistema más grande conocido como suprasistema (Kreps, 1995).

Tabla 1. Enfoque del modelo Holo.

\begin{tabular}{|l|l|}
\hline Pregunta & \multicolumn{1}{|c|}{ Justificación } \\
\hline $\begin{array}{l}\text { ¿Por qué desarrollar un modelo } \\
\text { con enfoque sistémico? }\end{array}$ & $\begin{array}{l}\text { Porque creemos que el enfoque sistémico es la forma correcta } \\
\text { de abordar la Ergonomía, Seguridad y Salud Ocupacional en } \\
\text { las organizaciones para alcanzar una mayor producción, con } \\
\text { más salud. }\end{array}$ \\
\hline ¿Para qué? & $\begin{array}{l}\text { Para detectar las desviaciones del sistema que lo apartan de un } \\
\text { mayor rendimiento y bienestar de sus integrantes y, por sobre } \\
\text { todo, para corregirlas. }\end{array}$ \\
\hline
\end{tabular}

De esta manera, la organización es visualizada como un sistema compuesto de seis subsistemas: organizacional, legal, salud, tecnológico/diseño, económico y social/comunidad.

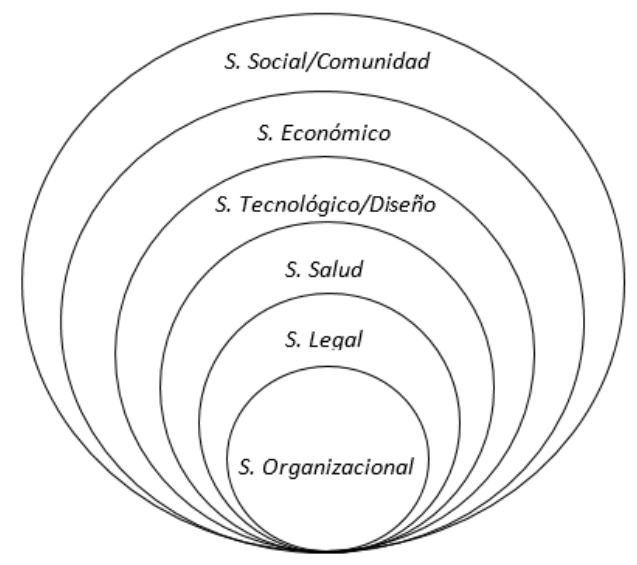

Figura 1. Sistema compuesto de seis subsistemas. 
A continuación, se define cada subsistema determinando cuáles son los componentes que se consideran de importancia en cada uno de ellos.

\section{Subsistema organizacional}

Este subsistema se relaciona con la cultura de la organización. Se entiende a la cultura organizacional como los "valores (qué es importante) y creencias (cómo funcionan las cosas) compartidos que al interactuar con las estructuras y sistemas de control de una organización producen normas de comportamiento (la manera como hacemos las cosas aquí)" (Reason, 2010).

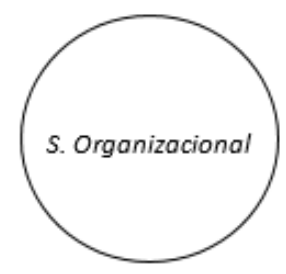

Figura 2. Subsistema organizacional.

\section{Subsistema legal}

Este subsistema está integrado por la legislación municipal, provincial y nacional en materia de seguridad y salud ocupacional (SySO) y medio ambiente (MA) que la organización debe cumplir. Así mismo, se considera dentro de este subsistema la relación de la organización con los otros actores del sistema de riesgos del trabajo: Ministerio de Trabajo, Empleo y Seguridad Social (MTEySS), Superintendencia de Riesgos del Trabajo (SRT), Aseguradoras de Riesgos del Trabajo (ART); y con los organismos reguladores en materia de Medio Ambiente como, por ejemplo: Organismo Provincial para el Desarrollo Sostenible (OPDS), Autoridad del Agua (ADA), etc.

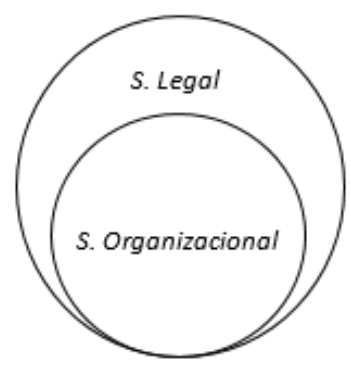

Figura 3. Subsistemas organizacional y legal.

\section{Subsistema salud}

Este subsistema está relacionado con las acciones concretas de los servicios o departamentos de SySO de la organización en materia de salud física, psíquica y social de sus integrantes (Organización Mundial de la Salud, 2014). 


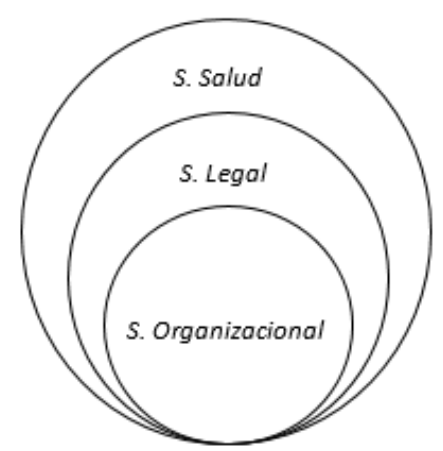

Figura 4. Subsistemas organizacional, legal y salud.

\section{Subsistema tecnológico/diseño}

El subsistema tecnológico/diseño tiene que ver con los procesos productivos llevados adelante por la organización y la tecnología que estos emplean.

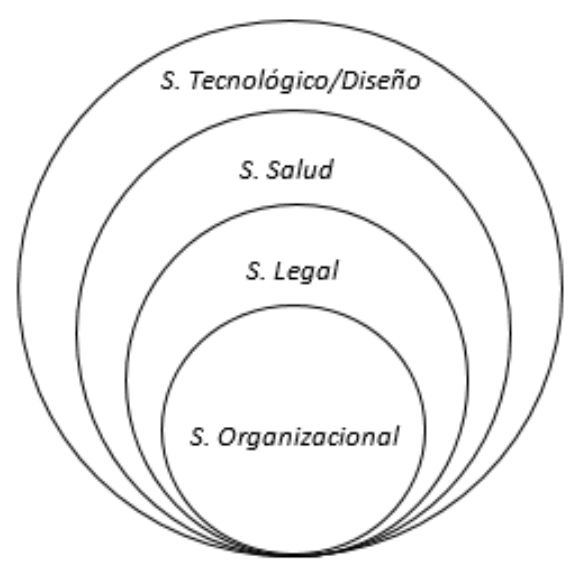

Figura 5. Subsistemas organizacional, legal, salud y tecnológico.

\section{Subsistema económico}

"La productividad puede ser comprendida sistemáticamente, como un equilibrio entre economía y salud" (García-Acosta, 2002). Dentro de este subsistema se encuentra la productividad de la organización, el empleo de insumos y materias primas, el tratamiento de los desperdicios; y la relación directa de estos elementos con los integrantes de la organización. 


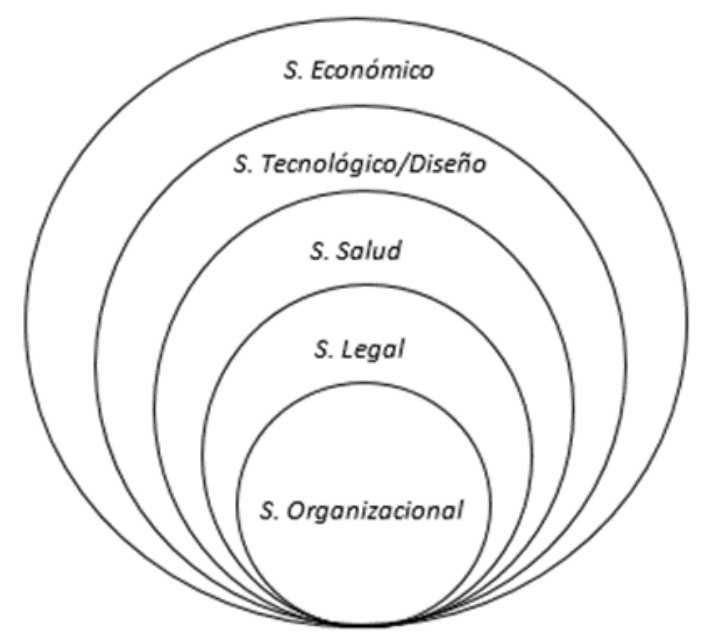

Figura 6. Subsistemas organizacional, legal, salud, tecnológico/diseño y económico.

\section{Subsistema social/comunidad}

El subsistema social/comunidad contempla la relación entre la organización y los miembros de la comunidad, localidad o provincia en la que se encuentra instalada; ya sean trabajadores, sus familias y otros habitantes.

Como ejes principales de este subsistema, se analiza la estrategia de incorporación de los trabajadores jóvenes y su desarrollo profesional. A su vez se aborda la temática del envejecimiento de la fuerza laboral, y la necesidad de contar con programas dinámicos de adecuación de sistemas de trabajo que consideren las limitaciones naturales provocadas por la edad. Incluye también los trabajadores migrantes, temática de género y trabajadores discapacitados.

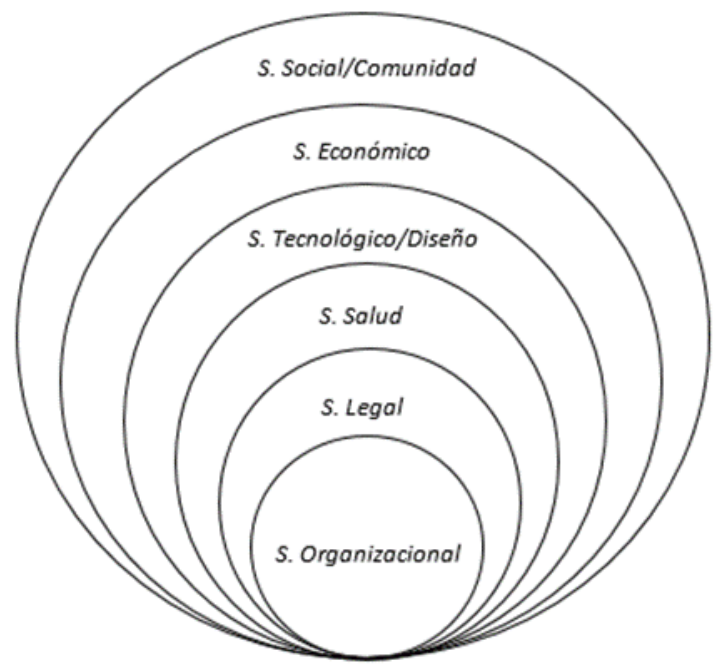

Figura 7. Subsistemas organizacional, legal, salud, tecnológico/diseño, económico y social/comunidad. 
El "Modelo Holos" se configura de la siguiente manera: los seis subsistemas que lo componen, los resultados de rendimiento y bienestar que cada sistema busca de forma permanente; los ergónomos junto con los equipos transdisciplinarios que serán los encargados de detectar y trabajar sobre las desviaciones del sistema que lo apartan del logro de los dos resultados mencionados. Al ergónomo y los equipos transdisciplinarios, se sumará la Universidad como soporte teórico, conceptual y de investigación, en caso que así se requiera.

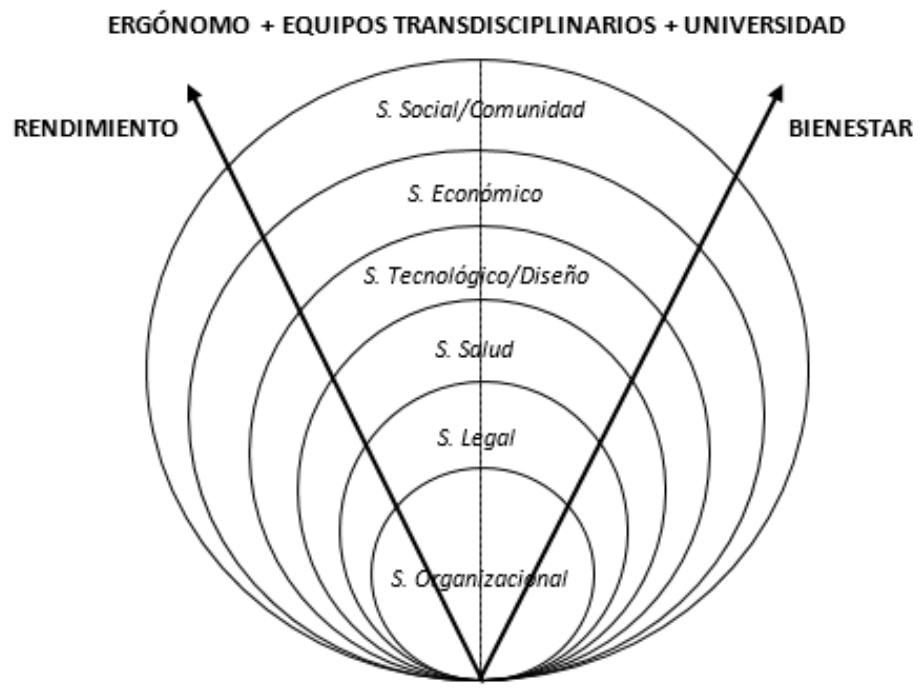

Figura 8. Modelo Holos.

\section{Relación de los subsistemas con los resultados esperados}

La intersección de cada subsistema con la línea del rendimiento representará la evaluación que se realice de cada uno de estos y de qué manera se apartan o contribuyen a la mejora del rendimiento de la organización. De la misma manera, la intersección de cada subsistema con la línea del bienestar representará la evaluación que se realice de cada uno de estos y de qué manera promueven o deterioran el bienestar de los integrantes de la organización. Se forman así, doce puntos de evaluación cualitativa del sistema. 


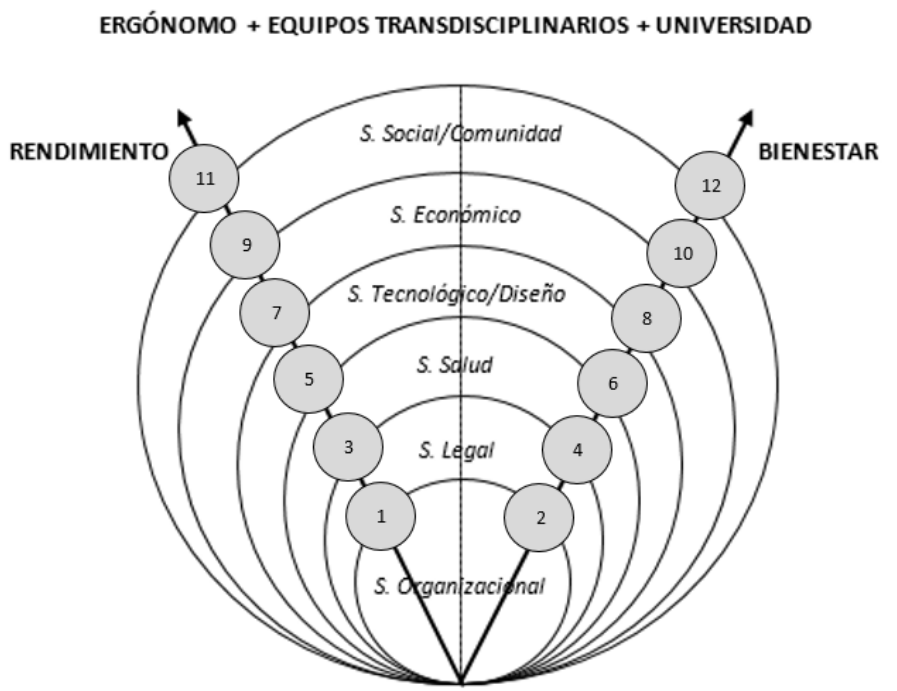

Figura 9. Relación de los subsistemas con los resultados esperado.

\section{Rol de los ergónomos y los equipos transdisciplinarios}

La intersección de cada subsistema con la línea punteada que se observa en la figura 10, representará la intervención de los ergónomos, primero para realizar la evaluación y el diagnóstico de cada uno de los doce puntos del modelo; y segundo, para realizar la corrección de los desvíos detectados. Será necesario para la aplicación del modelo, que los ergónomos trabajen de manera conjunta con los especialistas internos de la organización que tengan relación con cada subsistema (EQUIPO).

La "transdisciplinariedad" es definida como:

El nivel más complejo y eficiente de interacción entre diversas disciplinas con alto grado de cooperación y coordinación en base a objetivos comunes. A este nivel se logra construir un lenguaje hídrido y una epistemología nueva, es decir, se establece una visión estratégica común consolidada, mediante un proyecto de transformación consciente y creativa con metodologías alternativas viables y alto nivel de solución de problemas complejos [...]. (Luengo \& Martínez-Álvarez, 2018, p. 18). 


\section{ERGÓNOMO + EQUIPOS TRANSDISCIPLINARIOS + UNIVERSIDAD}

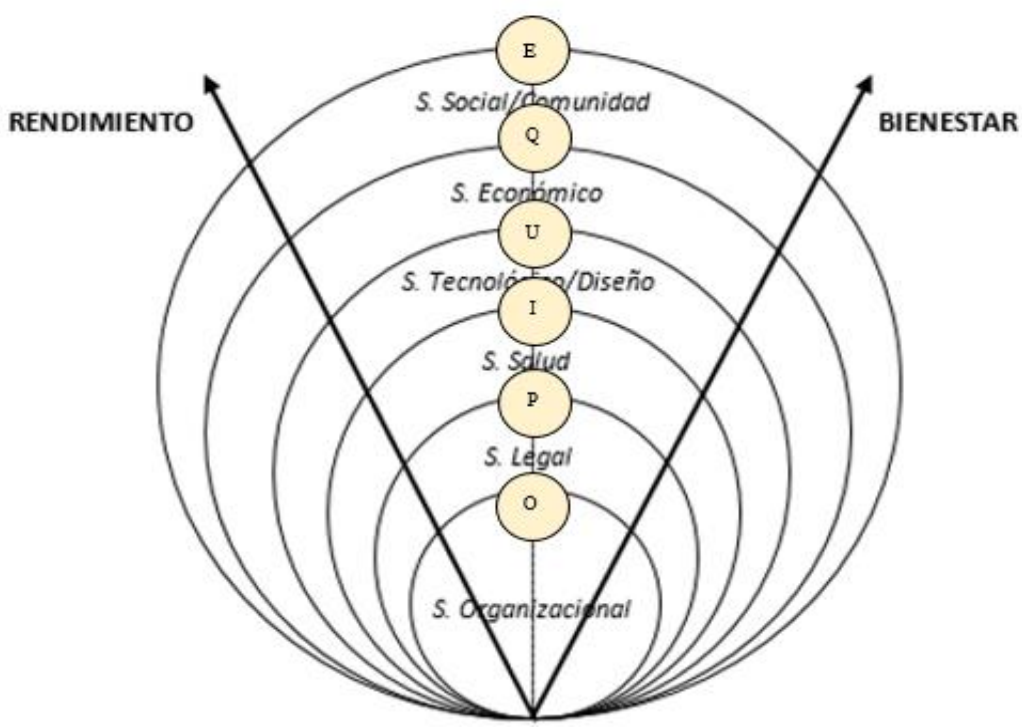

Figura 10. Rol de los ergónomos y los equipos transdisciplinarios.

\section{Análisis y evaluación fase I}

Cada uno de los doce puntos representa una serie de preguntas (108 en total) que harán el diagnóstico cualitativo y ofrecerán un panorama sobre la situación de la organización con respecto a cada subsistema.

Tabla 2. Apertura de los seis subsistemas en veintisiete elementos de análisis.

\begin{tabular}{|c|c|}
\hline Subsistema & Elementos de análisis \\
\hline Organizacional & $\begin{array}{l}\text { Organigrama - misión y visión - política - turnos - comunicación- } \\
\text { sindicato }\end{array}$ \\
\hline Legal & Matriz legal - capacitación - índices de siniestralidad - certificaciones \\
\hline Salud & $\begin{array}{l}\text { Servicio de higiene y seguridad - servicio de salud ocupacional - } \\
\text { ergonomía }\end{array}$ \\
\hline Tecnológico/diseño & $\begin{array}{l}\text { Procesos - automatización y robótica - digitalización y TIC - diseño y } \\
\text { usabilidad - mantenimiento - lay out }\end{array}$ \\
\hline Económico & Productividad - capital humano e intelectual \\
\hline Social/comunidad & $\begin{array}{l}\text { Trabajadores jóvenes - envejecimiento de la fuerza laboral - } \\
\text { trabajadores migrantes - discapacidad - equidad de género, } \\
\text { responsabilidad social empresaria y medio ambiente }\end{array}$ \\
\hline
\end{tabular}


De cada elemento, además del análisis cualitativo, se propone elaborar un escenario futuro. Se conformará además, un equipo (integrado por el ergónomo y los especialistas internos) por cada subsistema, que serán los responsables de obtener la información necesaria de la organización para responder cada pregunta. Una vez que se evalúe y cuantifique cada uno de los doce ítems, se obtendrá una valoración inicial de situación fase I.

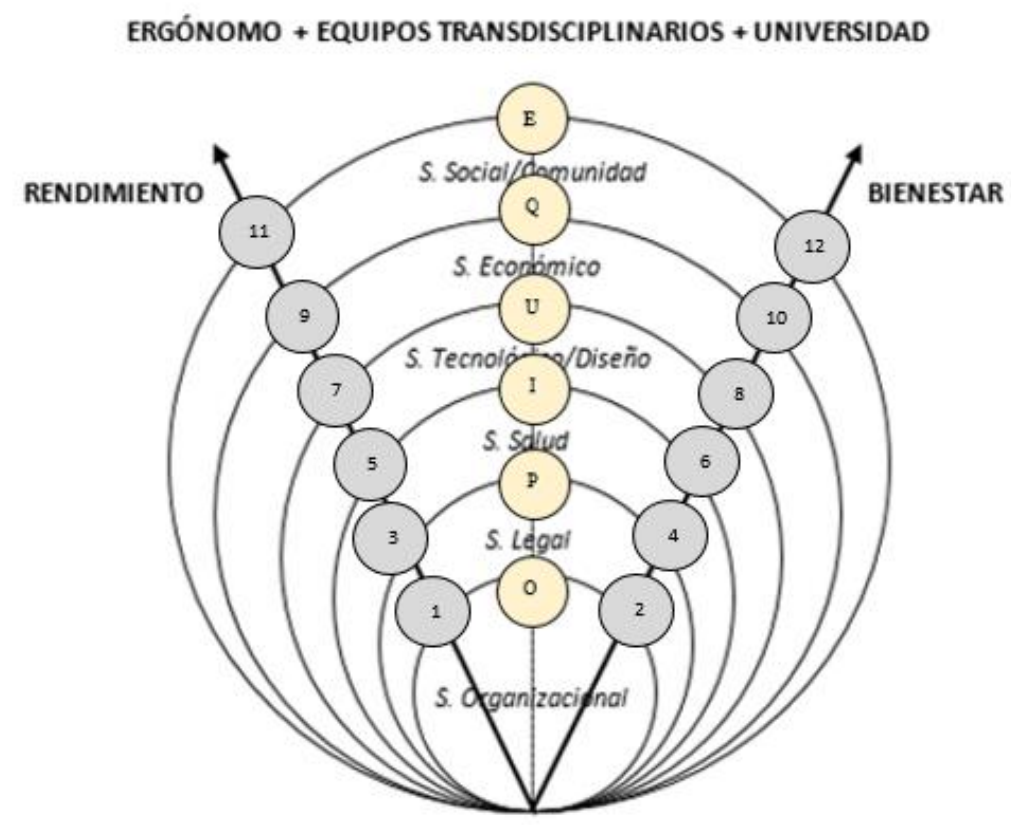

Figura 11. Análisis y evaluación.

\section{Conclusiones}

Con la aplicación del Modelo Holos se ratifican las características con las que fue pensado y creado. El modelo es descriptivo y cualitativo en su fase I (porque se asignan puntajes de acuerdo a la condición observada en la organización), metodológico (porque se aplica de acuerdo a una planificación de fases), prospectivo (especialmente en proponer una visión de futuro para cada elemento analizado), dinámico, y actualizable anualmente. En conclusión, es un modelo que aprende y se retroalimenta en su aplicación, pero además, enseña e informa en diversos temas a aquellos que lo aplican.

La fase I, que implica el análisis cualitativo de los seis subsistemas de forma independiente, fue llevada a cabo durante el año 2019. Una vez terminada esta fase, se obtuvieron cincuenta y una oportunidades de mejora, cuyas veinte más importantes para la gerencia, se aplicarán durante el 2020. En cuanto a la fase II basada en el análisis prospectivo, se pudo observar que muchos de los escenarios futuros también visualizados el año pasado ya fueron implementados debido a la necesidad de adaptación organizacional motivada por la pandemia. En estos momentos, se encuentra en desarrollo la fase III, determinando el grado de vulnerabilidad y resiliencia de la organización.

El nivel de recomendaciones surgidas de esta aplicación, tuvo impacto no sólo en la 
planta donde se realiza la intervención, sino que las mismas migrarán hacia las demás plantas de la firma, multiplicando en cantidad los beneficios obtenidos.

\section{Referencias}

Filippi, G. (2003). El aporte de la psicología del trabajo a los procesos de mejora organizacional. Buenos Aires: Editorial Universitario de Buenos Aires.

García-Acosta, G. (2002). La Ergonomía desde la visión sistémica. Bogotá: Editorial Universidad Nacional de Colombia, Universidad Nacional de Colombia.

Kreps, G. (1995). La comunicación en las organizaciones. USA: Edition Addison-Wesley.

Luengo, N. \& Martínez-Álvarez, F. (2018). La educación transdiciplinaria. (1a ed). Ciudad Autónoma de Buenos Aires: Editora Latinoamericana.

Organización Mundial de la Salud (2014). Documentos básicos. (48 a ed). Ginebra: Ediciones de la O.M.S.

Reason, J. (2010). La gestión de los grandes riesgos. Principios humanos y organizativos de la seguridad. Madrid: Modus Laborandi. 